2. To: (Receiving Organization)

LMHC/TWRS

East Tank Farms

5. Proj./Prog./Dept./Div.:

Project $W-314$

8. Originator Remarks:

Initial release of the Acceptance for Beneficial Use for the sections of Project $W-314$, Phase 1 listed below.

11. Receiver Remarks: 11A. Design Baseline Document? [] Yes [X] No
3. From: (Originating Organization) Tank Farm Upgrade Projects

6. Design Authority/ Design Agent/Cog. Engr.:

D. E. BOWERS
4. Related EDT No.:

$N / A$

7. Purchase Order No.:

$\mathrm{N} / \mathrm{A}$

9. Equip./Component No.:

$N / A$

10. System/Bldg./Facility:

N/A

12. Major Assm. Dwg. No.:

N/A

13. Permit/Permit Application No.: $N / A$

14. Required Response Date:

N/A

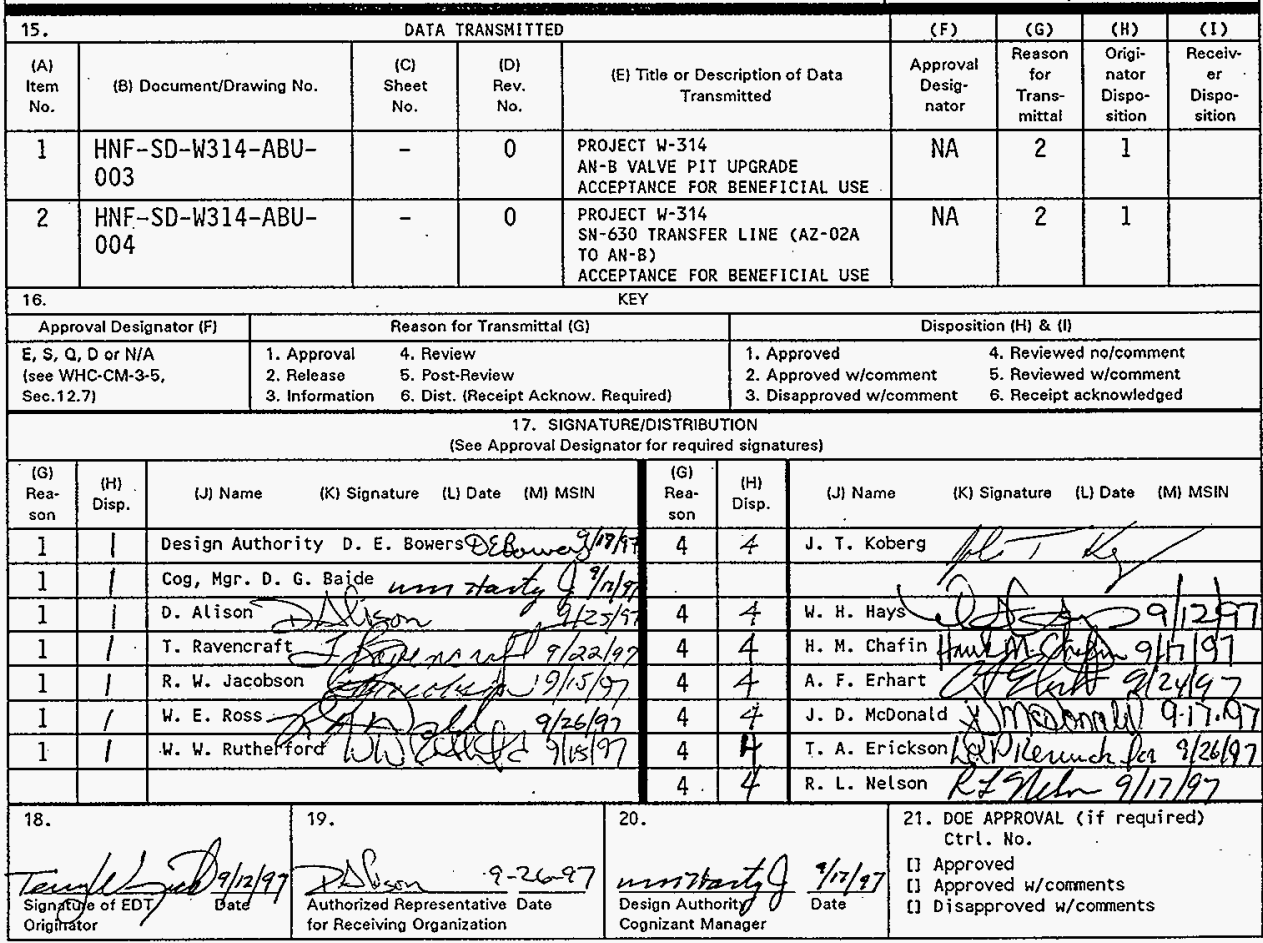

BD-7400-172-2 (05/96) GEF097 


\begin{tabular}{|c|c|c|c|}
\hline & $\begin{array}{l}\text { NGINEERING DATA TRA } \\
\text { CONTINUATION PAGE }\end{array}$ & & \\
\hline $\begin{array}{l}\text { 5. Proj./Prog./Dept./Div.: } \\
\text { Project W-314 }\end{array}$ & $\begin{array}{l}\text { 6. Cog. Eng. } \\
\text { D. E. Bowers }\end{array}$ & $\begin{array}{l}\text { 1. EDT } \\
615800\end{array}$ & Page 2 of 2 \\
\hline
\end{tabular}

\begin{tabular}{|c|c|c|c|c|c|c|c|c|}
\hline 15. & & DATA & ANSMITT & & (F) & (G) & (H) & (I) \\
\hline $\begin{array}{l}\text { (A) } \\
\text { Item } \\
\text { No. }\end{array}$ & (B) Document/Drawing No. & $\begin{array}{l}\text { (C) } \\
\text { Sheet } \\
\text { No. }\end{array}$ & $\begin{array}{l}\text { (D) } \\
\text { Rev. } \\
\text { No. }\end{array}$ & $\begin{array}{l}\text { (E) Title or Description of Data } \\
\text { Transmitted }\end{array}$ & $\begin{array}{l}\text { Approval } \\
\text { Desig- } \\
\text { nator }\end{array}$ & $\begin{array}{l}\text { Reason } \\
\text { for } \\
\text { Trans- } \\
\text { mittal }\end{array}$ & $\begin{array}{l}\text { Origi- } \\
\text { nator } \\
\text { Dispo- } \\
\text { sition }\end{array}$ & $\begin{array}{l}\text { Receiv- } \\
\text { or } \\
\text { Dispo- } \\
\text { sition }\end{array}$ \\
\hline 3 & $\begin{array}{l}\text { HNF-SD-W314-ABU- } \\
005\end{array}$ & - & 0 & $\begin{array}{l}\text { PROJECT } W-314 \\
\text { AX-8 VALVE PIT UPGRADE } \\
\text { ACCEPTANCE FOR BENEFICIAL USE }\end{array}$ & $\overline{N A}$ & 2 & 1 & \\
\hline 4 & $\begin{array}{l}\text { HNF-SD-W314-ABU- } \\
006\end{array}$ & - & 0 & $\begin{array}{l}\text { PROJECT W-314 } \\
\text { SN-632 IRANSFER LINE (AX-B TO } \\
\text { AZ-02A) } \\
\text { ACCEPTANCE FOR BENEFICIAL USE }\end{array}$ & NA & 2 & 1 & \\
\hline 5 & $\begin{array}{l}\text { HNF-SD-W314-ABU- } \\
007\end{array}$ & - & 0 & $\begin{array}{l}\text { PROJECT } \mathrm{W}-314 \\
\text { AZ-O2A PUMP P1T UPGRADE } \\
\text { ACCEPTANCE FOR BENEFICIAL USE }\end{array}$ & NA & 2 & 1 & \\
\hline 6 & $\begin{array}{l}\text { HNF-SD-W314-ABU- } \\
008\end{array}$ & - & 0 & $\begin{array}{l}\text { PROJECT } W-314 \\
\text { SN-634 TRANSFER LINE (AB TO } \\
\text { AX-B) } \\
\text { ACCEPTANCE FOR BENEFICIAL USE }\end{array}$ & NA & 2 & 1 & \\
\hline 7 & $\begin{array}{l}\text { HNF-SD-W314-ABU- } \\
009\end{array}$ & - & 0 & $\begin{array}{l}\text { PROJECT } W-314 \\
\text { AB VALVE PIT UPGRADE } \\
\text { ACCEPTANCE FOR BENEFICIAL USE }\end{array}$ & NA & 2 & 1 & \\
\hline & & & & & & & & \\
\hline & & & & & & & & \\
\hline & & & & & & & & \\
\hline & & & & & . & & & \\
\hline & & & & & & & & \\
\hline & & & & ? & & & & \\
\hline & & & & & & & & \\
\hline & & & & & & & & \\
\hline & & & & & & & & \\
\hline & & & & & & & & \\
\hline & & & & & & & & \\
\hline & & & & & & & & \\
\hline & & & & & & & & \\
\hline & & & & & & & & \\
\hline & & & & & & & & \\
\hline & & & & & & & & \\
\hline & & & & & & & & \\
\hline & & & & & & & & \\
\hline
\end{tabular}




\section{PROJECT W-314 \\ AX-B VALVE PIT UPGRADE Acceptance For Beneficial Use}

Terry Warnick

Fluor Daniel Northwest, Richland, WA 99352

U.S. Department of Energy Contract DE-AC06-96RL13200

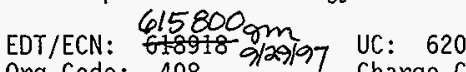

Org Code: 408

B\&R Code: EW3130010 Tota1 Pages: 12

Key Words: AX-B Valve Pit Upgrade, ABU

Abstract: $N / A$

TRADEMARK DISCLAIMER. Reference herein to any specific commercial product, process, or service by trade name, trademark, manufacturer, or otherwise, does not necessarily constitute or imply its endorsement, recommendation, or favoring by the United States Government or any agency thereof or its contractors or subcontractors.

Printed in the United States of America. To obtain copies of this document, contact: Document Control Services, P.O. Box 950, Mailstop H6-08, Richland WA 99352, Phone (509) 372-2420; Fax (509) 376-4989.
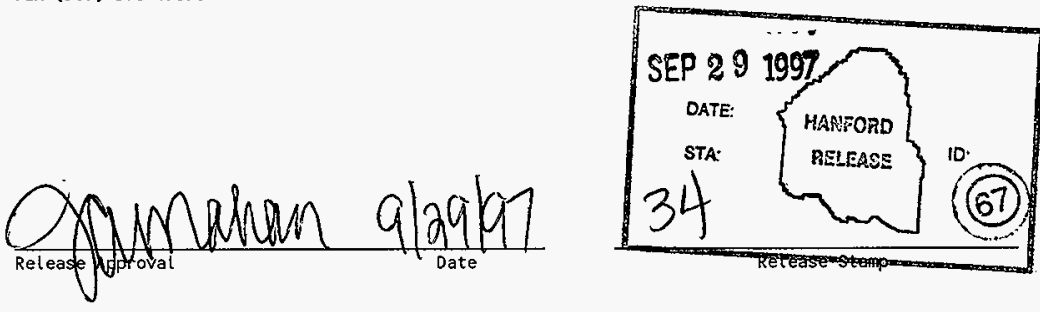
Program/Project Title: Project W-314, Tank Farm Restoration and Safe Operation, Phase 1

Component/System: AX-B Valve Pit Ungrade

September 15, 1997

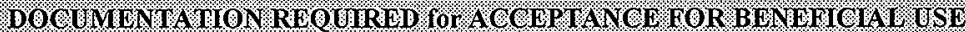

\begin{tabular}{|c|c|c|c|}
\hline DESCRIPTION & RESPONSIBILITY & DESCRIPTION & RESPONSIBILITY \\
\hline ENGINEERING & & ENGINEERING (cont'd) & \\
\hline [ ] Engineering Task Plan - N/A & & [X] Construction Specification & Rickenbach \\
\hline$[X]$ Activity Schedule & Rutherford & [ ] Essential Material Specification - N/A & \\
\hline [X] USQ Screening & Leach & [ ] lnstallation Work Plan - N/A & \\
\hline [ ] Final Safety Analysis Report - AB Amendment - N/A & & [X] Final Design Drawings & Rickenbach \\
\hline [ ] Interim Safety Basis - AB (BIO) Amendment - N/A & & [ ] Installation Drawings - N/A & \\
\hline I ] Safety Assessment - N/A & & {$[\mathrm{X}]$ As-Built Drawings $\mathrm{H}-2$} & Rickenbach \\
\hline$[\mathrm{X}]$ Safety Equipment List & Homan & [X] PCP Construction Inspection Records & Miller \\
\hline I ] Operational Safety Document(s) - N/A & & {$[X]$ IEFD Drawings - (P \& ID) } & Rickenbach \\
\hline [X] Design Criteria (Project Development Specification (PDS)) & Bowers & {$[\mathrm{X}]$ Systems Drawings H-14 (BIO, "Essential") } & Rickenbach \\
\hline [X] Requirements Verification Report for PDS & Rickenbach/Hays & {$[X]$ Drawing Tree/Index } & Rickenbach \\
\hline [ ] System Design Description-N/A & & {$[\mathrm{X}]$ As-Built Project Generated Drawings/ECNs } & Rickenbach \\
\hline$[\mathrm{X}]$ Project Design Concept (PDC) & Rickenbach & {$[X]$ Void Superseded Drawings } & Nelson \\
\hline$[X]$ Test Plan/Specifications (TEP) & Hays & [X] Master Equipment List Update & Nelson \\
\hline$[\mathrm{X}]$ Acceptance Test Procedures & Rickenbach & ADP (all computer activities are on MPS ABU) & \\
\hline$[X]$ Acceptance Test Report & Hays & [ ] Software Configuration Management Plan - N/A & \\
\hline$[\mathrm{X}]$ Operational Test Procedures & Hays & [ ] Systems Requirements Spec - N/A & \\
\hline [X] Operational Test Report & Hays & [ ] Software Design Description - N/A & \\
\hline [X] Environmental Impact Statement (TWRS) & Boes & [ I Software Validation/Verification - N/A & \\
\hline [ ] Environmental Report - N/A & & TRAINING & \\
\hline [ ] Environmental Permit - N/A & & {$[X]$ Training Plan } & Monris \\
\hline [ ] Hazardous Waste Disposal Plan/Procedures - N/A & & {$[\mathrm{X}]$ Training Manuals/Certification Package } & Morris \\
\hline [ ] Solid Waste Disposal Plan/Procedure - N/A & & {$[X]$ Training to Operating Crews } & Morris \\
\hline [X] Notice of Construction/Excavation & Boes & [X] Training to Maintenance Crews & Morris \\
\hline$[\mathrm{X}]$ Stress/Seismic Analysis & Rickenbach & {$[X]$ Training Mock-Up } & Hays \\
\hline [ ] Stress/Design Report - N/A & & QUALITY ASSURANCE & \\
\hline [ ] Design Specification Report - N/A & & {$[\mathrm{X}]$ Inspection Plans } & Chafin \\
\hline I ] Design Specifications/Report - N/A & & [X] QAPP & Chafin \\
\hline 1 ] Equipment Specifications - N/A & & I IQAPjP-N/A & \\
\hline$[\mathrm{X}]$ Procurement Specifications & Rickenbach & & \\
\hline
\end{tabular}


Program/Project Title: Project W-314, Tank Farm Restoration and Safe Operation, Phase 1

Component/System: AX-B Valve Pit Upgrade

September 29, 1997

\section{DOC UMENT ITON REOUIRED TOR AC CEPTANCE FOR BENETICIAL USE (cont d)}

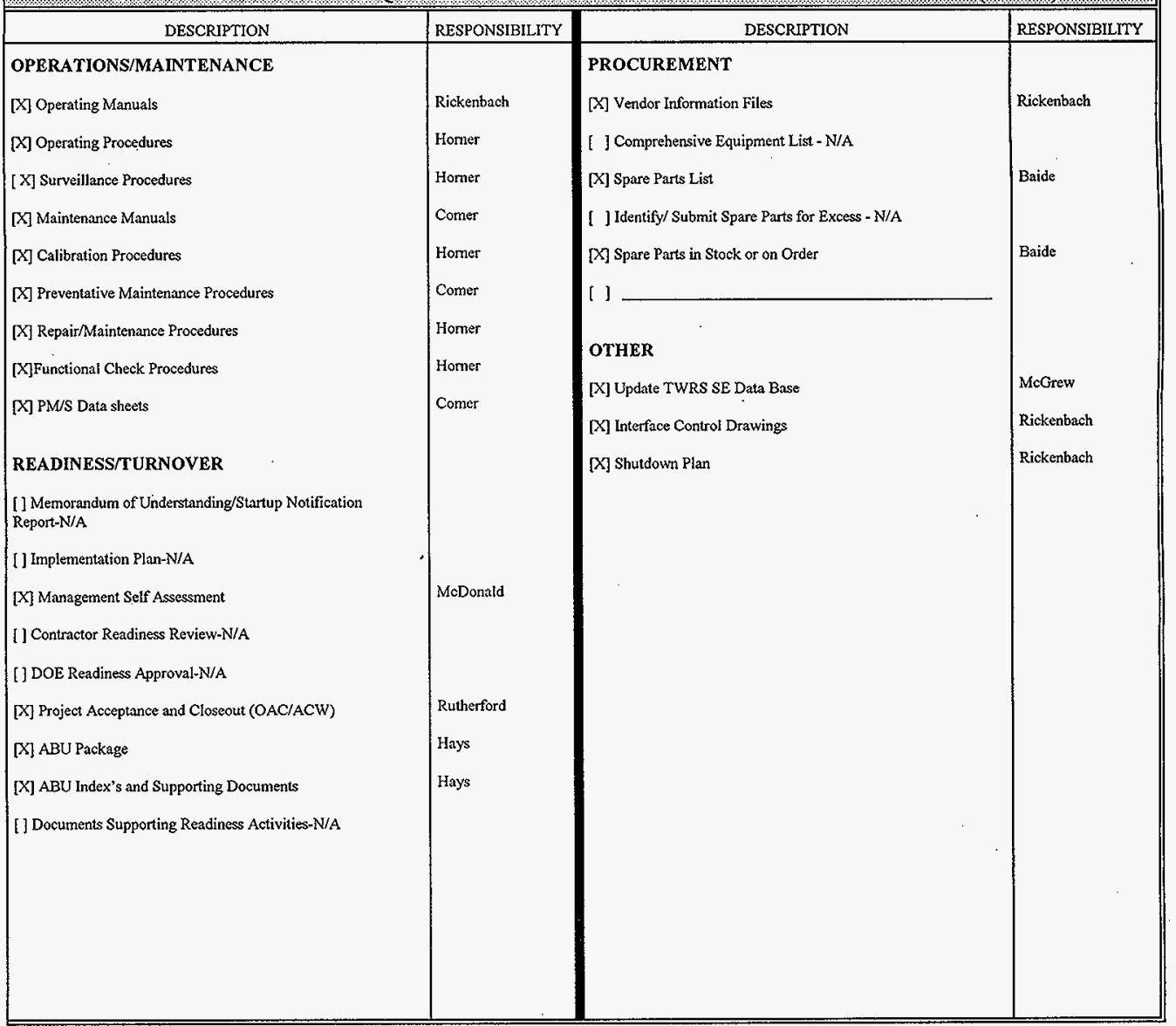




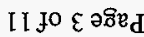

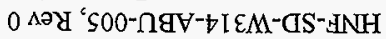

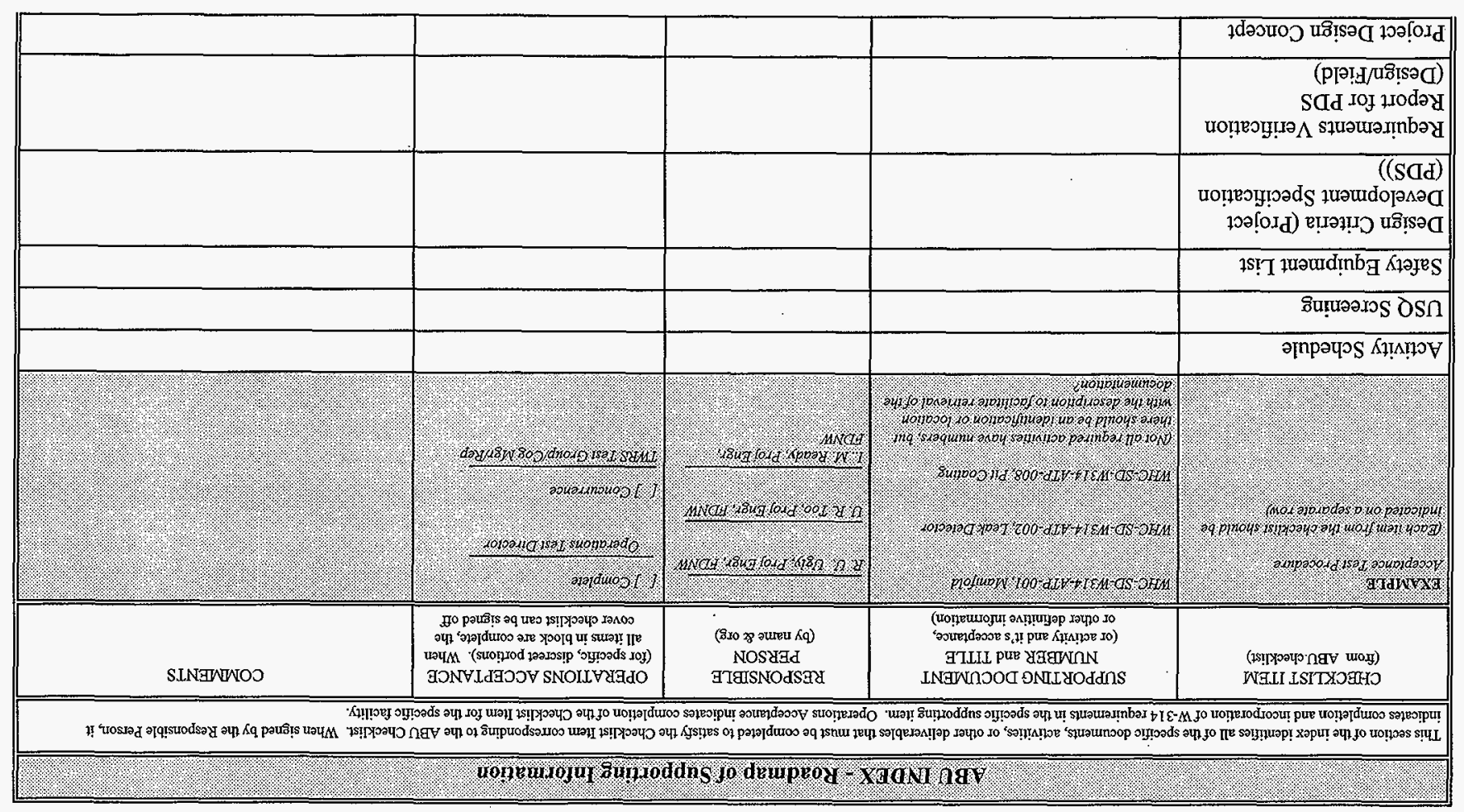

L66I 'I I rəqurə1dəS

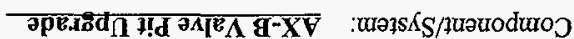

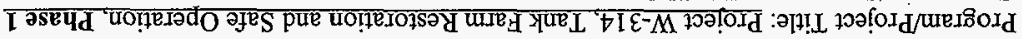


Program/Project Title: Project W-314, Tank Farm Restoration and Safe Operation, Phase 1

Component/System: AX-B Valve Pit Upgrade

September 11, 1997

\section{ABU INDEX - Roadinap of Supporting Information}

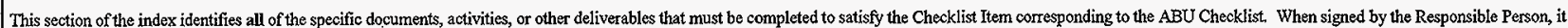
indicates completion and incorporation of W-314 requirements in the specific supporting item. Operations Acceptance indicates completion of the Checklist Item for the specific facility.

\begin{tabular}{|c|c|c|c|c|}
\hline $\begin{array}{l}\text { CHECKLIST ITEM } \\
\text { (from } \mathrm{ABU} \text { checklist) }\end{array}$ & $\begin{array}{l}\text { SUPPORTING DOCUMENT } \\
\text { NUMBER and TITLE } \\
\text { (or activity and it's acceptance, } \\
\text { or other definitive information) }\end{array}$ & $\begin{array}{c}\text { RESPONSIBLE } \\
\text { PERSON } \\
\text { (by name \& org) }\end{array}$ & $\begin{array}{l}\text { OPERATIONS ACCEPTANCE } \\
\text { (for specific, discreet portions). When } \\
\text { ail items in block are complete, the } \\
\text { cover checklist can be signed off }\end{array}$ & COMMENTS \\
\hline \multicolumn{5}{|l|}{$\begin{array}{l}\text { Test Plans/Specifications } \\
\text { (TEP) }\end{array}$} \\
\hline \multicolumn{5}{|l|}{$\begin{array}{l}\text { Acceptance Test } \\
\text { Procedures }\end{array}$} \\
\hline \multicolumn{5}{|l|}{ Acceptance Test Report } \\
\hline \multicolumn{5}{|l|}{$\begin{array}{l}\text { Operational Test } \\
\text { Procedures }\end{array}$} \\
\hline \multicolumn{5}{|l|}{ Operational Test Report } \\
\hline \multicolumn{5}{|l|}{$\begin{array}{l}\text { Environmental Impact } \\
\text { Statement (TWRS) }\end{array}$} \\
\hline \multicolumn{5}{|l|}{$\begin{array}{l}\text { Notice of } \\
\text { Construction/Excavation }\end{array}$} \\
\hline \multicolumn{5}{|l|}{ Stress/Seismic Analysis } \\
\hline \multicolumn{5}{|l|}{ Procurement Specification } \\
\hline \multicolumn{5}{|c|}{ Constructions Specification } \\
\hline \multicolumn{5}{|l|}{ Final Design Drawings } \\
\hline As-Build Drawings H-2 & & & & \\
\hline
\end{tabular}

HNF-SD-W314-ABU-005, Rev 0

Page 4 of 11 
Program/Project Title: Project W-314, Tank Farm Restoration and Safe Operation, Phase 1

Component/System: AX-B Valve Pit Upgrade

September 11, 1997

\section{ABU INDEX - Roadmap of Supporting Information}

This section of the index identifies all of the specific documents, activities, or other deliverables that must be completed to satisfy the Checklist Item corresponding to the ABU Checklist. When signed by the Responsible Person, it indicates completion and incorporation of W-314 requirements in the specific supporting item. Operations Acceptance indicates completion of the Checklist Item for the specific facility.

\begin{tabular}{|c|c|c|c|c|}
\hline $\begin{array}{l}\text { CHECKLIST ITEM } \\
\text { (from ABU checklist) }\end{array}$ & $\begin{array}{l}\text { SUPPORTING DOCUMENT } \\
\text { NUMBER and TITLE } \\
\text { (or activity and it's acceptance, } \\
\text { or other definitive information) }\end{array}$ & $\begin{array}{c}\text { RESPONSBBLE } \\
\text { PERSON } \\
\text { (by name \& org) }\end{array}$ & $\begin{array}{l}\text { OPERATIONS ACCEPTANCE } \\
\text { (for specific, discreet portions). When } \\
\text { all items in block are complete, the } \\
\text { cover checklist can be signed off }\end{array}$ & COMMENTS \\
\hline \multicolumn{5}{|l|}{$\begin{array}{l}\text { PCP Construction } \\
\text { Inspection Records }\end{array}$} \\
\hline \multicolumn{5}{|l|}{ IEFD Drawings- (P\&D) } \\
\hline \multicolumn{5}{|l|}{ Drawing Tree Index } \\
\hline \multicolumn{5}{|c|}{$\begin{array}{l}\text { As-Build Project Generated } \\
\text { Drawings/ECNs }\end{array}$} \\
\hline \multicolumn{5}{|c|}{ Void Superseded Drawings } \\
\hline \multicolumn{5}{|l|}{$\begin{array}{l}\text { Master Equipment List } \\
\text { Update }\end{array}$} \\
\hline \multicolumn{5}{|l|}{ Training Plan } \\
\hline \multicolumn{5}{|l|}{$\begin{array}{l}\text { Training } \\
\text { Manuals/Certification } \\
\text { Package }\end{array}$} \\
\hline \multicolumn{5}{|l|}{$\begin{array}{l}\text { Training to Operating } \\
\text { Crews }\end{array}$} \\
\hline $\begin{array}{l}\text { Training to Maintenance } \\
\text { Crews }\end{array}$ & & & & \\
\hline
\end{tabular}

HNF-SD-W3 14-ABU-005, Rev 0

Page 5 of 11 
I I fo 9 ว8в $\mathrm{d}$

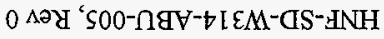

\begin{tabular}{|c|c|c|c|c|}
\hline & & & & 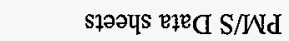 \\
\hline & & & & 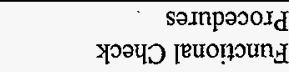 \\
\hline & & & & 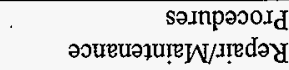 \\
\hline & & & & 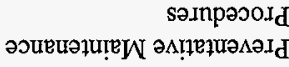 \\
\hline & & & & sə.Inpəoosd uo!teIque \\
\hline & & & & 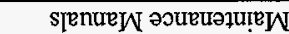 \\
\hline & & & & 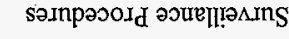 \\
\hline & & & & 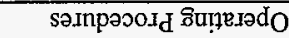 \\
\hline & & & & sienuew sumesado \\
\hline & & & & ddVO \\
\hline & & & & sueId uo!̣joadsu] \\
\hline & & & & 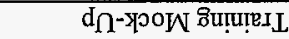 \\
\hline SINATKWOD & 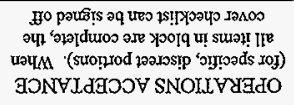 & 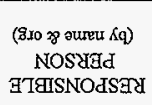 & 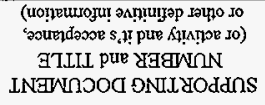 & 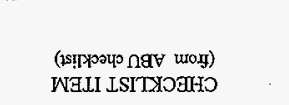 \\
\hline \multicolumn{5}{|c|}{ 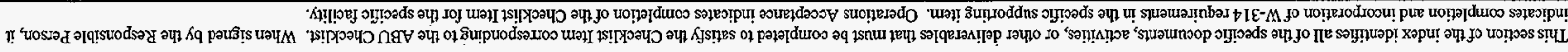 } \\
\hline \multicolumn{5}{|c|}{ 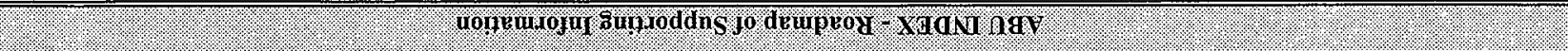 } \\
\hline
\end{tabular}

\section{L66I'I I Iəquә7dəS}

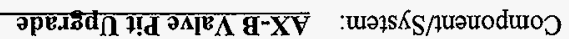

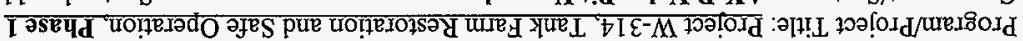


11 jo $L$ ว $8 e_{d}$

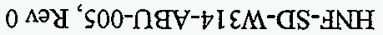

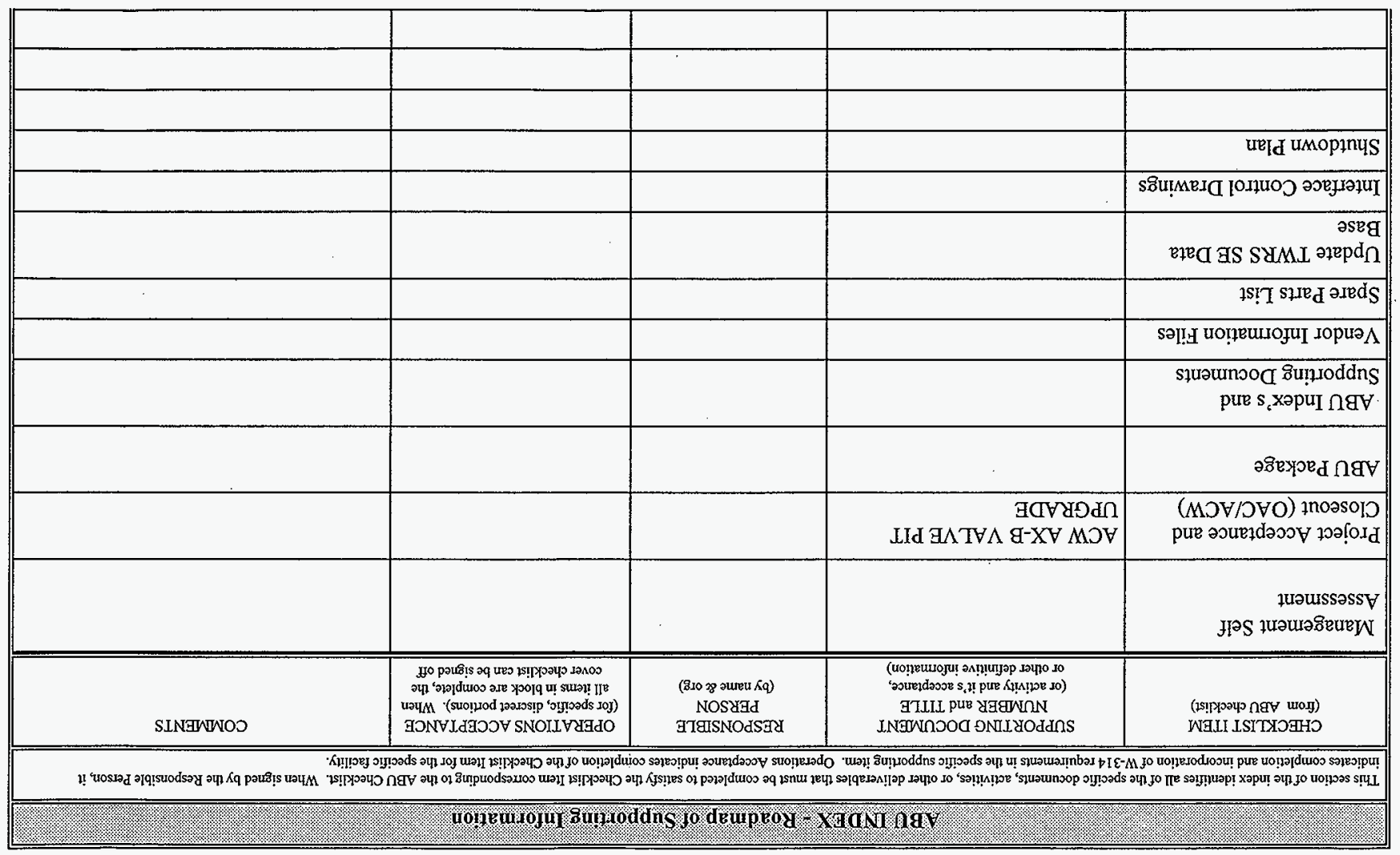

L66I 'EZ دəquə]dəs

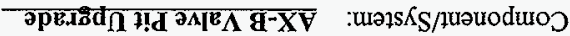

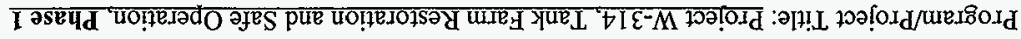


Program/Project Title: Project W-314, Tank Farm Restoration and Safe Operation, Phase 1

Component/System: AX-B Valve Pit Upgrade

September 11, 1997

\section{ABU INDEX - Roadmap of Supporting Information}

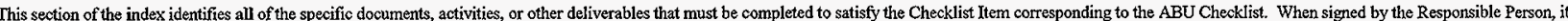
indicates completion and incorporation of W-314 requirements in the specific supporting item. Operations Acceptance indicates completion of the Checklist Item for the specific facility.

\begin{tabular}{||l|c|c|c|c|}
\hline \hline $\begin{array}{c}\text { CHECKLIST ITEM } \\
\text { (from ABU checklist) }\end{array}$ & $\begin{array}{c}\text { SUPPORTING DOCUMENT } \\
\text { NUMBER and TITLE } \\
\text { (or activity and it's acceptance, } \\
\text { or other definitive information) }\end{array}$ & $\begin{array}{c}\text { RESPONSTBLE } \\
\text { PERSON } \\
\text { (by name \& org) }\end{array}$ & $\begin{array}{c}\text { OPERATIONS ACCEPTANCE } \\
\text { (for specific, discreet portions). When } \\
\text { all items in block are complete, the } \\
\text { cover checklist can be signed off }\end{array}$ & \\
\hline \hline & & & & \\
\hline End of Table & & & & \\
\hline
\end{tabular}




\section{ABU NDEX - Acceptance Documentation}

This section of the index identifies Operations acceptance of individual segments for turnover. When signed, Operations has accepted completion of the specific segment, with Design Authority concurrence. The final signatures at the end of this index reflect Operations acceptance of all operational, maintenance, and administrative responsibilities for the structures, systems, and components associated with this $A B U$.

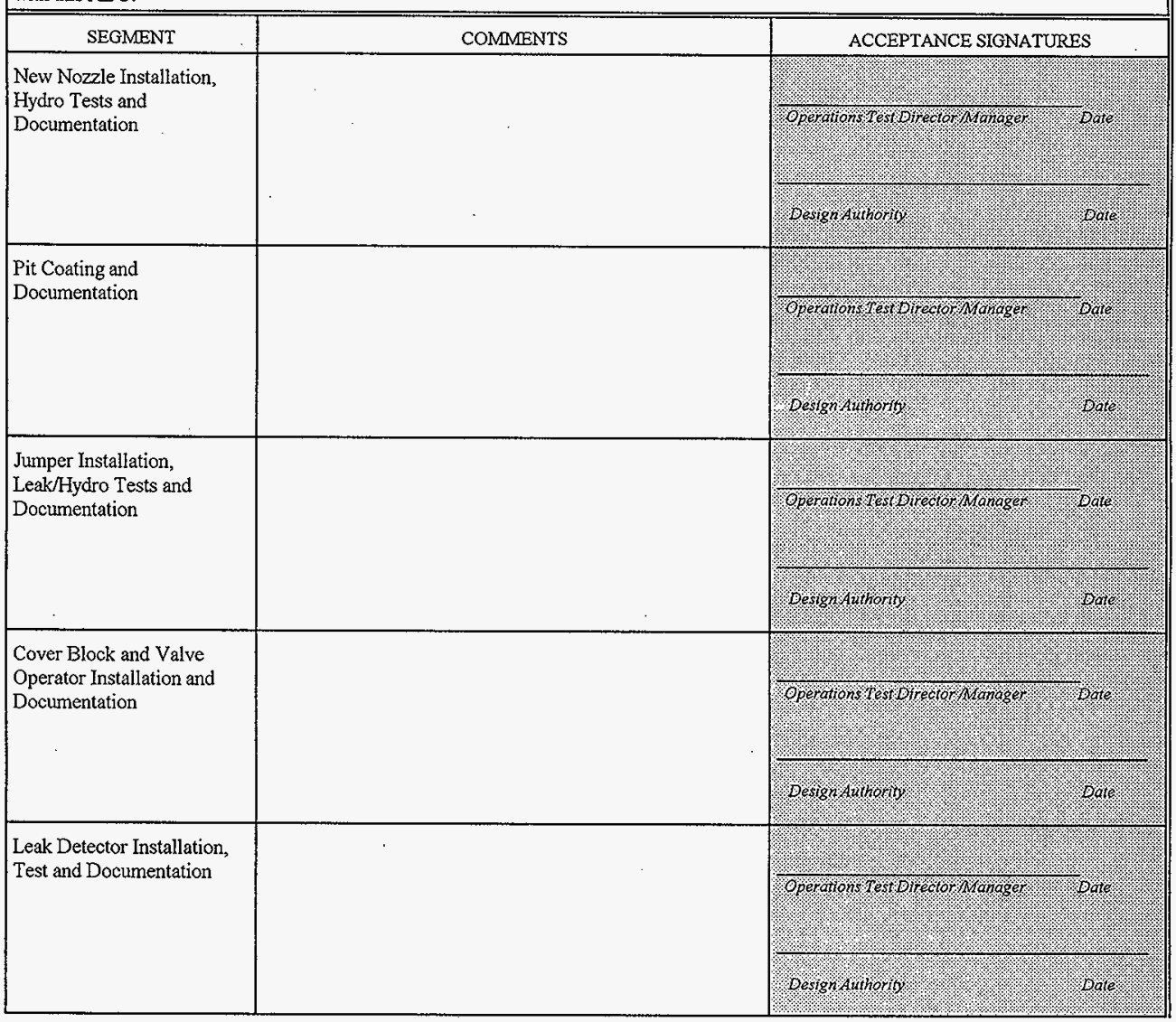


Program/Project Title: Project W-314, Tank Farm Restoration and Safe Operation, Phase 1

Component/System: $\underline{\text { AX-B Valve Pit Upgrade }}$

September 23, 1997

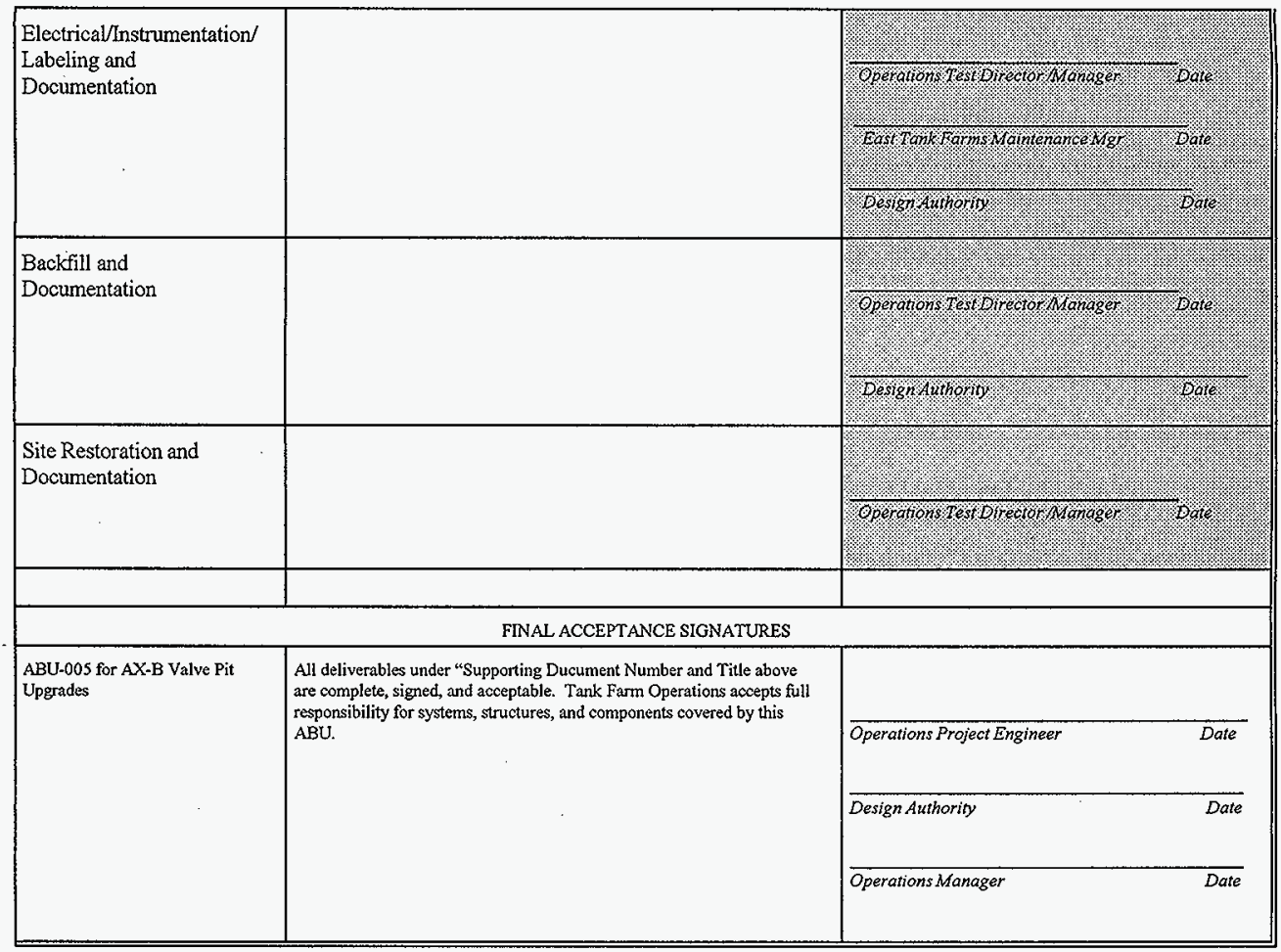


Program/Project Title: Project W-314, Tank Farm Restoration and Safe Operation, Phase 1 Component/System: $\underline{\text { AX-B Valve Pit Upgrade }}$

\section{ABU INDEX - Post Acceptance Punch List}

This section of the index identifies items still requiring completion or closure after acceptance of the segment/system per the ABU. Note: Items affecting Readiness may not be on the punchlist without documented justification, an approved plan to correct the deficiency, and written concurrence from the Director of Tank Farms (or his delegee).

\begin{tabular}{|c|c|c|c|}
\hline ITEM & $\begin{array}{c}\text { RESPONSIBLE PERSON } \\
\text { (Signature) } \\
\text { AND ORGANZATION }\end{array}$ & $\begin{array}{l}\text { COMMT } \\
\text { MENT } \\
\text { DATE }\end{array}$ & ACCEPTANCE OF COMPLETION \\
\hline & & & \\
\hline & & & \\
\hline & & & \\
\hline & & & \\
\hline & & & \\
\hline & & & \\
\hline & & & \\
\hline & & & \\
\hline & & & \\
\hline & & & \\
\hline & & & \\
\hline & & & \\
\hline & & & \\
\hline & & & \\
\hline & & & \\
\hline & & & \\
\hline End of Table & & & \\
\hline
\end{tabular}




\section{DISTRIBUTION SHEET}

\begin{tabular}{|c|c|c|c|c|c|}
\hline To & \multirow{2}{*}{\multicolumn{2}{|c|}{$\begin{array}{l}\text { From } \\
\text { Terry Warnick }\end{array}$}} & & \multicolumn{2}{|c|}{ Page 1 of 1} \\
\hline Distribution & & & & \multicolumn{2}{|c|}{$\begin{array}{l}\text { Date September 12, } \\
1997\end{array}$} \\
\hline \multirow{2}{*}{$\begin{array}{l}\text { Project Title/Work Order } \\
\text { Project } W-314 \text { PHASE } 1 \text { ABUS }\end{array}$} & & \multirow[t]{2}{*}{. } & & \multicolumn{2}{|c|}{ EDT No. 615800} \\
\hline & & & & \multicolumn{2}{|c|}{ ECN No. NA } \\
\hline Name & MSIN & $\begin{array}{c}\text { Text } \\
\text { With All } \\
\text { Attach. }\end{array}$ & Text Only & $\begin{array}{l}\text { Attach./ } \\
\text { Appendix } \\
\text { Only }\end{array}$ & $\begin{array}{l}\text { EDT/ECN } \\
\text { Only }\end{array}$ \\
\hline
\end{tabular}

D. E. Bowers

D. G. Baide

W. M. Harty, Jr

D. Alison

T. Ravencraft

R. W. Jacobson

W. E. Ross

W. W. Rutherford

T. A. Erickson

3. T. Koberg

W. H. Hays

H. M. Chafin

A. F. Erhart

J. D. McDonald

R. 1. Neison

C. E. Leach

J. L. Homan

M. D. Rickenbach

K. A. Boes

D. R. Miller

J. M. Morris

T. M. Horner

J. W. Comer

D. L. McGrew

W. H. Bryant

R. 3. Shupe

B. L. Syverson
S5-13

55-05

S5-13

S5-03

S5-01

R1-09

S5-07

R3-25

R2-50

G3 -12

E6-08

R3-25

R2-50

R2 -50

R3 -47

R1-49

Rl-43

G3-12

R3-25

G3-12

R2-87

R2-82

R2-88

R3-25

SO-09

R2-50

H5- 57 $x$

$X$

$x$

$x$

$x$

$X$

$X$

$x$

$x$

$x$

$x$

$\hat{X}$

$\hat{x}$

$x$

$X$

$X$

$x$

$x$

$X$

$x$

$x$

$X$

$X$

$x$

$x$

$X$

$\hat{X}$ 